\title{
THE COMPATIBILITY POLYMORPHISM IN INVERTEBRATE HOST/TREMATODES INTERACTIONS: RESEARCH OF MOLECULAR DETERMINANTS
}

\author{
BOUCHUT A.*, ROGER E.*, GOURBAL B.*, GRUNAU C.*, COUSTAU C.** \& MITTA G.*
}

\section{Summary :}

The co-evolutionary dynamics that exist in many host-parasite interactions sometimes leads to compatibility polymorphism. This phenomenon is well documented in mollusc/trematodes interactions but its molecular base is unknown. In order to identify key molecules involved in this phenomenon, we developed several molecular approaches comparing compatible or incompatible strains of mollusc or parasite. These comparisons led to the identification of numerous candidate genes listed and discussed (some of them) in the present review.

KEY WORDS : Biomphalaria glabrata, Schistosoma mansoni, Echinostoma caproni, compatibility polymorphism.

T The comprehensive understanding of host-parasite interactions represents a major challenge in evolutionary biology. Because parasites are responsible for substantial deleterious effects, they represent a major driving force for the evolution of their hosts. In parallel, parasites have to co-evolve with their host to avoid elimination. This adaptation of the Red Queen hypothesis to host-parasite systems (Combes, 2000) predicts that an arms race will lead to the potential antagonistic evolution of host resistance and parasite virulence processes leading to the co-evolution of host-parasite systems. In some models, parasite virulence and host defence are in equilibrium in natural populations. This is the case in host-parasite systems for which only certain host and parasite phenotypes are compatible. Such systems constitute models of choice due to the fact that their co-evolutionary dynamics are experimentally accessible permitting the use of comparative approaches between compatible and incompatible strains to identify the molecules playing a key role in the interaction.

The models we have chosen to study are the interaction between the trematodes Echinostoma caproni or Schistosoma mansoni, the agent of human intestinal

\footnotetext{
* Parasitologie Fonctionnelle et Evolutive, UMR 5244, CNRS Université de Perpignan, France.

** Inserm, U 547, Université Lille 2, Institut Pasteur de Lille, IFR 142, France.

Correspondence: Guillaume Mitta, UMR 5244, CNRS Université de Perpignan, 52, avenue Paul Alduy, 66860 Perpignan Cedex, France. Tel.: 33 (0)4686621 88 - Fax: 33 (0)4 68662281.

E-mail: mitta@univ-perp.fr
}

schistosomiasis (Gryseels et al., 2006) and their invertebrate intermediate host, the gastropod mollusc Biomphalaria glabrata. In these interactions, a compatibility polymorphism has been evidenced and permits us to select compatible and incompatible strains on which comparative molecular approaches are applicable. In addition, the comparison between these two parasites sharing the same host is interesting because their immune evasion strategy seems to be completely different (Loker \& Adema, 1995). Evidences strongly support the hypothesis that the major immune evasion strategy of $S$. mansoni relies on a molecular mimicry mechanism and would therefore avoid recognition as non-self invader by the snail immune system. In contrast to S. mansoni, echinostomes are known to strongly interfere with the snail immune response via their excretory-secretory (ES) products (Humbert \& Coustau, 2001).

The present review focus on the different works we have published the three last years in these two different systems.

\section{THE INTERACTION BETWEEN B. GLABRATA AND E. CAPRONI}

Th then n this model B. glabrata/E. caproni, two snail strains of $B$. glabrata were selected in our laboratory (Langand et al., 1998). E. caproni miracidia are able to penetrate both compatible (susceptible) and incompatible (resistant) snails. While the parasite undergoes normal development in compatible snails, in incompatible snails it is encapsulated and eliminated. Susceptibility/resistance mechanisms of B. glabrata to E. caproni are probably inherited in a multigenic fashion (Langand \& Morand, 1998) and have been shown to rely on both humoral and cellular factors (Ataev \& Coustau, 1999). In vivo comparison of E. caproni development in both $B$. glabrata strains revealed that, in resistant snails, sporocysts were abnormally developed and degenerated regardless of their level of encapsulation (Ataev \& Coustau, 1999). These results suggested that humoral factors are actively involved in the molecular processes 
underlying resistance or susceptibility to E. caproni (Ataev \& Coustau, 1999). As a first approach to investigate the molecular bases of this phenotype difference between strains, we used comparative proteomics with plasma collected from both strains. We revealed 13 plasmatic proteins exhibited significant differences in their apparent representativity (Vergote et al., 2005). The cDNAs corresponding to five of these proteins were identified. They encode proteins belonging to three different classes. The first is a type- 2 secreted cystatin that was named Bg type- 2 cystatin. The cystatins are cysteine protease inhibitors that control activity of cathepsins, themselves involved in various processes, including immunity (Abrahamson et al., 2003). Two others are isoforms of secreted Calcium Binding proteins of the EF-hand type (Bg CaBP 1 and 2) that are known to play key role in calcium homeostasis. And the two last proteins are two isoforms of an endo-1,4-mannanase that are known to randomly cleave within the $\beta$-1,4-mannan main chain of galactomannan, glucomannan, galactoglucomannan and mannan (Xu et al., 2002). All these proteins are secreted, more represented in the plasma of susceptible snails and these differences appear correlated with transcript levels (Vergote et al., 2005). In addition, analysis of their expression in a range of tissues showed that they are expressed by the albumen gland (Vergote et al., 2005). Post-infection expression studies of these five candidate genes showed that the transcript content of all candidates are stable in susceptible snails following infection, while they increase significantly in exposed resistant snails (Vergote et al., 2005). All the differences observed between susceptible and resistant snails could be linked to a differential regulation of genes expressed by the albumen gland. In molluscs, the albumen gland is considered as an accessory sexual gland (Wijdenes et al., 1983). Nevertheless, this organ is also involved in the production of defense effectors like agglutinins in planorbid snails (Michelson \& Dubois, 1977; Stein \& Basch, 1979; Boswell \& Bayne, 1984) or antimicrobial proteins in oposthobranchs gastropods (Iijima et al., 1994; Takamatsu et al., 1995). Our results further support the idea that the albumen gland may play a significant role in snail immune response and raise the question of the potential importance of this organ in processes underlying susceptibility/resistance to E. caproni.

Regarding the cellular factors, previous studies showed that excretory-secretory (ES) products from in vitro transformed E. caproni sporocysts inhibited key defence functions of susceptible host hemocytes such as adhesion and phagocytosis (Humbert \& Coustau, 2001). Interestingly, hemocytes from resistant snails remained unaffected by these parasite ES products, suggesting that they exhibit differences compared with hemocytes from susceptible snails (Humbert \& Coustau, 2001). We develop two comparative approaches in order to disco- ver the molecular basis of this phenotype difference. Because such differences were indirectly or directly related to adhesive properties of hemocytes, we developed a first targeted approach to examine the expression of genes directly involved in adhesion processes (Bouchut et al., 2006). In this study, we compared the transcript content of all known hemocytic genes potentially involved in adhesion processes between hemocytes of the two strains. Transcripts of four genes were differentially present between hemocytes from resistant and susceptible snails. These genes encode proteins ressembling dermatopontins (two isoforms), matrilin and cadherin (Bouchut et al., 2006). Among them, dermatopontins seem to be promising candidates. These novel dermatopontins (Bg dermatopontin 2 and -3) are probably secreted and the structural features of the precursors suggest that these two molecules share, in addition to dermatopontin general characteristics, some other particular features already observed in two other invertebrate dermatopontins from Suberites domuncula (cysteine array, (Schutze et al., 2001)) and Limulus polyphemus (additionnal internal repeat, (Fujii et al., 1992)). The function of these particular structural features has not been elucidated. Nevertheless, it was shown that these two dermatopontins triggered adhesive processes of dissociated cells from $S$. domuncula (Schutze et al., 2001) or hemocytes from L. polyphemus (Fujii et al., 1992). These data suggest that $B$. glabrata hemocytic dermatopontins could participate in aggregation processes of hemocytes. Futhermore, we showed in the same work (Bouchut et al., 2006) that the transcript content of these two genes was: $i$ ) higher in hemocytes from unexposed resistant snails (in comparison with those of susceptible snails, and $i$ ) greatly enhanced in resistant snails $48 \mathrm{~h}$ after exposure to the parasite. Interestingly, previous histological studies showed that, in E. caproni-exposed resistant snails, the hemocytic capsule was mounted at 48/72 h post exposure (Ataev \& Coustau, 1999). The fact that the higher level of expression of $\mathrm{Bg}$ dermatopontins observed in resistant snails at $48 \mathrm{~h}$ after exposure coincides with the capsule formation, further supporting the hypothesis that this gene could play a key role in adhesive processes taking place during capsule formation in resistant snails.

After this first targeted transcriptomic approach developed on hemocytes of both strains, we developed a global proteomic approach (Bouchut et al., 2006). The comparative approach we used to study hemocytes collected from susceptible and resistant snails revealed that 12 out of the 2,552 proteins from hemocytes exhibited significant differences in their apparent abundance. Direct comparison of tags obtained by LC-MSMS to databases allowed the identification of five of these 12 differentially represented proteins. These proteins were named $\mathrm{Bg}$ aldolase, $\mathrm{Bg}$ intermediate filament protein, $\mathrm{Bg}$ cytidine deaminase, $\mathrm{Bg}$ ribosomal protein $\mathrm{P} 1$ 
and Bg histone H4 (see (Bouchut et al., 2006) for discussion of their putative function).

These different approaches taken together allowed identification of several candidate genes and suggested a potential involvement of tissues other than hemocytes in susceptibility/resistance processes (Vergote et al., 2005; Bouchut et al., 2006). In order to investigate this latter point, we compared the transcripts of the two snail strains at the whole body level using a Suppression Subtractive Hybridization (SSH) approach (Bouchut et al., 2007). This approach revealed several genes identified in the above mentioned studies but also new candidates belonging to novel functional groups of interest. The functional groups of - glycolytic enzymes and Calcium Binding Proteins (CaBP) - were identified from $B$. glabrata plasma in the above mentioned proteomic approach (Vergote et al., 2005) and were also identified by SSH. Regarding novel functional groups, the most promising candidates identified in the present study belong to immunity class and were identified in the library containing genes over expressed in resistant snails. For example, we identified a cluster displaying similarities for a defence factor Aplysianin A purified from the albumen gland of Aplysia kurodai (Takamatsu et al., 1995). This glycoprotein inhibited the growth of both Gram-positive and -negative bacteria. Another study developed in Aplysia punctata has evidenced a molecule called APIT (A. punctata Ink Toxin) displaying about $60 \%$ identity to Aplysianin A (Butzke et al., 2005). APIT is an L-amino acid oxidase. This enzyme produces hydrogen peroxide and was shown to lead to a necrosis-like oxidative damage of eukaryotic cells. The Bg Aplysianin A-like we identify in the SSH study could be a defence factor involved in the antiparasitic response of $B$. glabrata. Its higher abundance in resistant snails could explain the efficiency of resistant snail response against E. caproni.

\section{THE INTERACTION BETWEEN B. GLABRATA AND S. MANSONI}

A nother model we have chosen to study in our laboratory is the interaction between $S$. mansoni, the agent of human intestinal schistosomiasis (Gryseels et al., 2006) and its invertebrate intermediate host, the gastropod mollusc B. glabrata. In this interaction, a compatibility polymorphism has been also evidenced (Theron \& Coustau, 2005). In compatible interactions, the parasite penetrates and develops normally within the snail and in incompatible interactions, the larval trematode penetrates but is recognized as nonself, encapsulated and destroyed by the mollusk's internal defense system. The success or failure of an infection does not depend on the snail susceptibi- lity/resistance status, but on the "matched" or "mismatched" status of the host and parasite phenotypes ("matching phenotype" hypothesis (Theron \& Coustau, 2005)).

In this $S$. mansoni/B. glabrata interaction, two $S$. mansoni strains are available, one compatible (strain C) and the other incompatible (strain IC) towards the same snail strain. Strain IC miracidia are immediately recognized as non-self and encapsulated in the first hours following penetration (Roger et al., 2007). The compatible ones remain unaffected. These observations suggest constitutive antigenic differences between strains. To identify and characterize the underlying molecular determinants, we conducted a global comparative proteomics approach on primary sporocysts (Sp1) from C and IC strains (Roger et al., 2007). This approach led us to identify a promising candidate belonging to a family of schistosome antigens that share some characteristics with the molecules of the mucin family (named Sm PoMucs). In particular, they display a domain containing a variable number of tandem repeats (VNTR) rich in S, $\mathrm{T}$ and $\mathrm{P}$. In a complementary work, we examine precursor structure and report the analysis of expression and polymorphism of this mucin-like family (Roger et al., 2007). The corresponding precursors are composed of three distinct domains: a signal peptide of 22 aminoacids followed by the variable domain containing a variable number of 9-residue tandem repeats and a Cterminal domain of 234 amino-acids. Three types of repeats were identified and named $r 1, r 1$ and $r 2$. Three groups of Sm PoMucs cDNAs were identified in both strains. Each group contains cDNAs that are identical in the deduced amino-acid sequence of the 234residue C-terminal domain. Extensive analysis of polymorphism revealed a crucial difference within and between the IC and C strains. The polymorphism revealed within the two first groups consists in a variable number of $\mathrm{r} 2$ repeats ( 1 to 55 ). The major difference between strains is observed for the third group of $\mathrm{Sm}$ PoMuc. This latter displays a VNTR domain containing only r1 and/or r1' in strain C. In the strain IC, this VNTR combination was observed, but interestingly, a strainspecific subgroup of molecular variants containing both repeats $\mathrm{r} 2$ and $\mathrm{r} 1 / \mathrm{r} 1$ ' was also identified.

This VNTR region of $S m$ PoMuc is of particular interest since in-silico prediction identifies this region as a potential target of glycosylation and we showed that Sm PoMuc proteins are indeed highly glycosylated. The VNTR region is therefore an excellent candidate for polymorphic glycosylation. Differences in the repeat type and number in the molecular variants could be responsible for differences in glycosylation status.

The polymorphism of $\mathrm{Sm}$ PoMucs is evidenced at different levels. The first level is due to differences in repeat number within the VNTR region. This phenomenon was observed previously for numerous mucins 
from protozoa and helminths (Hicks et al., 2000; Theodoropoulos et al., 2001), but also for other parasitic molecules displaying a VNTR region. Examples are the SOWgp cell surface proteins from the pathogenic fungi Coccidioides immitis (Hung et al., 2000) and different proteins from Plasmodium, the circomsporozoite proteins (CSP, (Nussenzweig \& Nussenzweig, 1985)) or the merozoite surface proteins (Msp-1 and Msp-2, (Holder, 1988; Smythe et al., 1988)). In the case of Plasmodium proteins, the repetitive regions seem to be not glycosylated but they are, as in the case of $S m$ PoMuc, highly polymorphic (Rich et al., 1997; Rich \& Ayala, 2000) and VNTR regions are present. Polymorphism generated by VNTR is well documented and new alleles can arise by duplication/deletion of the repeated DNA sequences through recombination involving unequal crossing-over (Rich et al., 1997). Our data suggest that a similar process operates to generate the variability of $\mathrm{Sm}$ PoMuc. The second level of polymorphism is unique to $\mathrm{Sm}$ PoMuc. We demonstrated a combinatory polymorphism specific of the IC strain. In order to understand this phenomenon, Sm PoMucs genomic structure analysis is underway.

Expression analysis of Sm PoMuc (Roger et al., 2007) strengthens the potential of these molecules as key determinants of the compatibility polymorphism in S. mansoni/B. glabrata interaction. Indeed, Sm PoMuc genes are only transcribed in stages interacting with molluscs with a peak of expression in the mollusc infective stage, the miracidium. Furthermore, peptide signal prediction indicates the possible secretion of these molecules. This hypothesis was strengthened by: i) immunolocalization of $S m$ PoMuc in the apical gland, and ii) the detection of $S m$ PoMuc in the ES products by western blotting. Our data show that these molecules are released in the immediate environment of the miracidia and Sp1. The apical gland is an organ involved in producing secretions necessary for the successful penetration of miracidia into snail tissue (Dresden et al., 1983; Yoshino et al., 1993) and is known to persist for a number of days after miracidium to sporocyst transformation in vitro (Basch \& DiConza, 1974; Wippersteg et al., 2002) and in vivo (Pan, 1980; Pan, 1996). The gland cells could therefore play a role in infestation and post-infestation process in the first hours following the contact between the host and its parasite. The rapid recognition, encapsulation and killing of the IC strain could be explained by the differences evidenced by our studies and we hypothesize that these differences could be responsible for recognition or non-recognition in the case of the IC and C strains, respectively.

Parasite-derived mucin or mucin-like molecules have been extensively described in different protozoan and helminth parasites, but their function has not yet been completely elucidated. They could have roles in host recognition, penetration, adhesion and invasion of host cells, immunoprotection, immunomodulation and in the avoidance of host immune processes (Hicks et al., 2000; Theodoropoulos et al., 2001). Among these molecules described in parasites, the mucin-like molecules of Trypanosoma cruzi have been extensively studied and share numerous characteristics with $\mathrm{Sm}$ PoMuc (see (Buscaglia et al., 2006) for review), such as their structure, a high level of glycosylation and polymorphism. A major difference is that these molecules encoded by the TcMUC I and II genes, like other surface components of parasitic protozoa, are typically anchored to the outer phospholipid layer of the plasma membrane by GPI. Nevertheless, secreted mucins have been characterized in helminths like the infective larvae of the parasitic nematode Toxocara canis (Loukas et al., 2000). These latter secreted mucins might simply create an immunological smoke-screen by generating antigenantibody complexes away from the parasite (Marin et al., 1992). An attractive hypothesis could be that Sm PoMucs could also create an immunological smokescreen able to block pattern recognition receptors, thus avoiding recognition and the host immune reaction. However, compatibility polymorphism evidenced in our system could alternatively be based on a lock and key mechanism involving Sm PoMucs and host recognition partner molecules. In this case positive recognition of the compatible variable antigen would determine success of the infection. Consequently, to further investigate this hypothesis, the potential partners of Sm PoMucs will be investigated using co-immunoprecipitation experiments. Diversified host molecules like FREPS (Adema et al., 1997; Zhang et al., 2004) could be ideal candidates. The data obtained in the two last papers presented here describe novel mucin-like proteins from $S$. mansoni. These Sm PoMucs are very attractive candidates to explain the compatibility polymorphism occuring in our system. Studies on gene structure, genomic organization and functional characterization are underway and will shed light on the exact role of these proteins in the infection of invertebrate hosts.

\section{CONCLUSION AND PERSPECTIVE}

The different comparative molecular approaches developed in our different experimental systems have highlighted several promising candidates. Nevertheless, these different approaches have also probably revealed genes that are not key determinants of the compatibility we are interested in. Indeed, several of these genes are probably strain markers co-selected with our key genes of interest. The challenge now is to develop functional studies aiming at the selection of actor genes of the compatibility. This challenge requires the development of different methodologies 
and tools not available in our systems. This is the challenge in our research field that is at the hour of genomics (genome sequencing and assembly of $S$. mansoni and B. glabrata) and is not yet entered in post-genomic time.

\section{REFERENCES}

Abrahamson M., Alvarez-Fernandez M. \& Nathanson C.M. Cystatins. Biochem. Soc. Symp., 2003, 179-199.

Adema C.M., Hertel L.A., Miller R.D. \& Loker E.S. A family of fibrinogen-related proteins that precipitates parasitederived molecules is produced by an invertebrate after infection. Proceedings of the National Academy of Sciences of USA, 1997, 94, 8691-8696.

Ataev G.L. \& Coustau C. Cellular response to Echinostoma caproni infection in Biomphalaria glabrata strains selected for susceptibility/resistance. Developmental and Comparative Immunology, 1999, 23, 187-198.

Basch P.F. \& DiConza J.J. The miracidium-sporocyst transition in Schistosoma mansoni: surface changes in vitro with ultrastructural correlation. Journal of Parasitology, 1974, 60, 935-941.

Boswell C.A. \& BAyne C.J. Isolation, characterization and functional assessment of a hemagglutinin from the plasma of Biomphalaria glabrata, intermediate host of Schistosoma mansoni. Developmental and Comparative Immunology, 1984, 8, 559-568.

Bouchut A., Coustau C., Gourbal B. \& Mitta G. Compatibility in the Biomphalaria glabrata/Echinostoma caproni model: new candidate genes evidenced by a suppressive subtractive hybridization approach. Parasitology, 2007, 134, 575-188

Bouchut A., Roger E., Coustau C., Gourbal B. \& Mitta G. Compatibility in the Biomphalaria glabrata/Echinostoma caproni model: potential involvement of adhesion genes. International Journal for Parasitology, 2006, 36, 175-184.

Bouchut A., Sautiere P.E., Coustau C. \& MitTa G. Compatibility in the Biomphalaria glabrata/Echinostoma caproni model: Potential involvement of proteins from hemocytes revealed by a proteomic approach. Acta Tropica, 2006, 98, 234-246.

Buscaglia C.A., Campo V.A., Frasch A.C. \& Di Noia J.M. Trypanosoma cruzi surface mucins: host-dependent coat diversity. Nature Reviews Microbiology, 2006, 4, 229-236.

Butzke D., Hurwitz R., Thiede B., Goedert S. \& Rudel T. Cloning and biochemical characterization of APIT, a new 1amino acid oxidase from Aplysia punctata. Toxicon, 2005 , 46, 479-89.

COMBEs C. Selective pressure in host-parasite systems. Journal de la Société de Biologie, 2000, 194, 19-23.

Dresden M.H., Sung C.K. \& DeElder A.M. A monoclonal antibody from infected mice to a Schistosoma mansoni egg proteinase. Journal of Immunology, 1983, 130, 1-3.

Fuji N., Minetti C.A., Nakhasi H.L., Chen S.W., Barbehenn E., NunEs P.H. \& NGuYen N.Y. Isolation, cDNA cloning, and characterization of an $18-\mathrm{kDa}$ hemagglutinin and amebocyte aggregation factor from Limulus polyphemus. Journal of Biological Chemistry, 1992, 267, 22452-22459.

Gryseels B., Polman K., Clerinx J. \& Kestens L. Human schistosomiasis. Lancet, 2006, 368, 1106-1118.

Hicks S.J., Theodoropoulos G., Carrington S.D. \& Corfield A.P. The role of mucins in host-parasite interactions. Part I-protozoan parasites. Parasitology Today, 2000, 16, 476-481.

HOlder A.A. The precursor to major merozoite surface antigens: structure and role in immunity. Progress in Allergy, 1988, 41, 72-97.

Humbert E. \& Coustau C. Refractoriness of host haemocytes to parasite immunosuppressive factors as a putative resistance mechanism in the Biomphalaria glabrata-Echinostoma caproni system. Parasitology, 2001, 122, 651-660.

Hung C.Y., Ampel N.M., Christian L., Seshan K.R. \& Cole G.T. A major cell surface antigen of Coccidioides immitis which elicits both humoral and cellular immune responses. Infection and Immunity, 2000, 68, 584-593.

Ijjima R., Kisugi J. \& YamaZaKi M. Biopolymers from marine invertebrates. XIV. Antifungal property of Dolabellanin A, a putative self-defense molecule of the sea hare, Dolabella auricularia. Biological Pharmaceutical Bulletin, 1994, 17, 1144-1146.

Langand J., Jourdane J., Coustau C., Delay B. \& Morand S. Cost of resistance, expressed as a delayed maturity, detected in the host-parasite system Biomphalaria glabrata/Echinostoma caproni. Heredity, 1998, 80, 320-325.

LANGAND J. \& MORAND S. Heritable non-susceptibility in an allopatric host-parasite system: Biomphalaria glabrata (Mollusca)-Echinostoma caproni (Platyhelminth, Digenea). Journal of Parasitology, 1998, 84, 739-742.

LOKER E.S. \& ADEMA C.M. Schistosomes, Echinostomes and Snails: comparative Immunobiology. Parasitology Today, 1995, 11, 120-124.

Loukas A., Hintz M., Linder D., Mullin N. P., Parkinson J., TetтeH K.K. \& Maizels R.M. A family of secreted mucins from the parasitic nematode Toxocara canis bears diverse mucin domains but shares similar flanking six-cysteine repeat motifs. Journal of Biological Chemistry, 2000, 275, 39600-39607.

Marin M.S., Prieto M., Martin J.M., Casais R., Boga J.A. \& PARRA F. Identification and expression of a Fasciola hepatica gene encoding a gut antigen protein bearing repetitive sequences. Molecular and Biochemical Parasitology, 1992, 55, 155-165.

Michelson E.H. \& Dubois L. Agglutinins and lysins in the molluscan family Planorbidae: a survey of hemolymph, eggmasses, and albumen-gland extracts. Biological Bulletin, 1977, 153, 219-227.

NussenzWEIG V. \& NussenzWEIG R.S. Circumsporozoite proteins of malaria parasites. Cell, 1985, 42, 401-403.

PAN S.C. The fine structure of the miracidium of Schistosoma mansoni. Journal of Invertebrate Pathology, 1980, 36, 307372.

PAN S.C. Schistosoma mansoni: the ultrastructure of larval morphogenesis in Biomphalaria glabrata and of associated host-parasite interactions. Japanese Journal of Medical Science and Biology, 1996, 49, 129-149. 
Rich S.M. \& Ayala F.J. Population structure and recent evolution of Plasmodium falciparum. Proceedings of the National Academy of Sciences of USA, 2000, 97, 6994-7001.

Rich S.M., Hudson R.R. \& Ayala F.J. Plasmodium falciparum antigenic diversity: evidence of clonal population structure. Proceedings of the National Academy of Sciences of USA, 1997, 94, 13040-13045.

Roger E., Gourbal B.E., Grunau C., Pierce R., Galinier R. \& MitTa G. Expression analysis of highly polymorphic mucin proteins (Sm PoMuc) from the parasite Schistosoma mansoni. Molecular and Biochemical Parasitology, 2007 (in press).

Roger E., Mitta G., Mone Y., Bouchut A., Rognon A., Grunau C., Boissier J., Theron A. \& Gourbal B.E. Molecular determinants of compatibility polymorphism in the Biomphalaria glabrata/Schistosoma mansoni model: new candidates identified by a global comparative proteomic approach. Molecular and Biochemical Parasitology, 2007 (in press).

Schutze J., Skorokhod A., Muller I.M. \& Muller W.E. Molecular evolution of the metazoan extracellular matrix: cloning and expression of structural proteins from the demosponges Suberites domuncula and Geodia cydonium. Journal of Molecular Evolution, 2001, 53, 402-415.

Smythe J.A., Coppel R.L., Brown G.V., Ramasamy R., Kemp D.J. \& ANDERS R.F. Identification of two integral membrane proteins of Plasmodium falciparum. Proceedings of the National Academy of Sciences of USA, 1988, 85, 5195-5199.

STEIN P.C. \& BASCH P.F. Purification and binding properties of hemagglutinin from Biomphalaria glabrata. Journal of Invertebrate Pathology, 1979, 33, 10-18.

Takamatsu N., Shiba T., Muramoto K. \& Kamiya H. Molecular cloning of the defense factor in the albumen gland of the sea hare Aplysia kurodai. FEBS Letters, 1995, 377, 373-376.

Theodoropoulos G., Hicks S.J., Corfield A.P., Miller B.G. \& CARRINGTON S.D. The role of mucins in host-parasite interactions: Part II - helminth parasites. Trends in Parasitology, 2001, 17, 130-135.

Theron A. \& Coustau C. Are Biomphalaria snails resistant to Schistosoma mansoni? Journal of Helminthology, 2005, 79, 187-191.

Vergote D., Bouchut A., Sautiere P.E., Roger E., Galinier R., Rognon A., Coustau C., Salzet M. \& Mitta G. Characterisation of proteins differentially present in the plasma of Biomphalaria glabrata susceptible or resistant to Echinostoma caproni. International Journal for Parasitology, 2005, 35, 215-224.

WijDENES J., VAN ElK R. \& JoOsSE J. Effects of two gonadotropic hormones on polysaccharide synthesis in the albumen gland of Lymnaea stagnalis, studied with the organ culture technique. General and Comparative Endocrinology, 1983, 51, 263-271.

Wippersteg V., Kapp K., Kunz W. \& Grevelding C.G. Characterisation of the cysteine protease ER60 in transgenic Schistosoma mansoni larvae. International Journal for Parasitology, 2002, 32, 1219-1224.

Xu B., Hagglund P., Stalbrand H. \& Janson J.C. Endo-beta1,4-Mannanases from blue mussel, Mytilus edulis: purifica- tion, characterization, and mode of action. Journal of Biotechnology, 2002, 92, 267-277.

Yoshino T.P., Lodes M.J., Rege A.A. \& Chappell C.L. Proteinase activity in miracidia, transformation excretory-secretory products, and primary sporocysts of Schistosoma mansoni. Journal of Parasitology, 1993, 79, 23-31.

Zhang S.M., Adema C.M., Kepler T.B. \& Loker E.S. Diversification of Ig superfamily genes in an invertebrate. Science, 2004, 305, 251-254. 